\title{
Die Open-Source-Software OpenBib an der USB Köln - Überblick und Entwicklungen in Richtung OPAC 2.0
}

\author{
Oliver Flimm <flimm@ub.uni-koeln.de>
}

13.4.2007

\begin{abstract}
Dieser Artikel beschreibt die vom Autor entwickelte und an der Universitätsund Stadtbibliothek Köln (USB) seit 1997 in verschiedenen Projekten eingesetzte Open-Source-Portalsoftware OpenBib. Nach einem grundlegenden Einblick in die Ursprünge, Technik und konkreten Einsatzgebiete an der USB werden weitergehende Funktionen des Portals erläutert, wie RSS-Feeds, Mashups, Tagging, Tag-Clouds, Nutzungsanalysen, Drilldowns sowie Kataloganreicherung. Viele davon sind charakteristisch für einen OPAC 2.0.
\end{abstract}

The Open Source software OpenBib at the University Library of Cologne (USB) General overview and developments towards OPAC 2.0

This article describes the Open Source portal software OpenBib, a project that was started by the author in 1997 and is being used in different projects at the University Library of Cologne (USB). After a basic overview of the origins, technology and the application at the USB the article describes some additional features such as RSS feeds, mashups, tagging, tag clouds, use analyses, drilldowns as well as catalogue enrichment. Many of these are characteristic for OPAC 2.0.

Le logiciel Open Source OpenBib à la Bibliothèque de I'Université de Cologne (USB) - Vue d'ensemble et développements vers la direction d'un OPAC 2.0

Cet article décrit le logiciel de portail Open Source OpenBib, développé par l'auteur et utilisé à la Bibliothèque de l'Université de Cologne (USB) depuis 1997 en différents projets. Après un regard élémentaire vers les origines, la technique et les domaines d'application concrets à l'USB, des fonctions additionelles du portail sont décrites, comme des flux RSS, des mashups, l'utilisation des étiquettes, des nuages de mots-clés, des analyses d'utilisation, des drilldowns ainsi qu'un enrichissement du catalogue. Beaucoup d'en sont caractéristiques pour un OPAC 2.0.

O. Flimm, Die Open-Source-Software OpenBib an der USB Köln - Überblick und Entwicklungen in Richtung OPAC 2.0 in: BIBLIOTHEK. Forschung und Praxis, Jg. 31 (2007) Nr. 2 


\section{Inhaltsverzeichnis}

1 KUG und OpenBib 2

1.1 Entkopplung von Portal und Bibliothekssystemen . . . . . . . . . . . . . . 4

1.2 Flexibilität durch Open-Source-Software . . . . . . . . . . . . . 5

$\begin{array}{|ll|}2 & \text { Ursprung und Technik von OpenBib }\end{array}$

2.1 Kaskadierung von Templates, CSS und Texten . . . . . . . . . . . . . . . 6

2.2 Recherche-Backends, UTF8-Encoding und I18N . . . . . . . . . . . . . . . 7

2.3 Administration und Betrieb . . . . . . . . . . . . . . . . . . . . . 7

$\begin{array}{lll}3 & \text { Einsatz in Fach-Portalen } & 8\end{array}$

4 Anreicherungen, Mashups und OPAC 2.0 9

4.1 Ergebnisanreicherung aller KUG-Datenbanken durch gescannte Inhaltsverzeichnisse ............................ 11

4.2 Individuelles und gemeinschaftliches Indexieren („Tagging“

4.3 Tag-Clouds als Orientierungshilfe . . . . . . . . . . . . . . . . . . . . . . . . 13

4.4 Auswertung des Nutzungsverhaltens . . . . . . . . . . . . . . . . . . . . . . . . 15

4.5 Drilldowns in Treffermengen . . . . . . . . . . . . . . . . . . . . . . . . . . . . 16

4.6 RSS-Feeds . . . . . . . . . . . . . . . . . . . . . . . . . . . . . . 16

4.7 Mashups . . . . . . . . . . . . . . . . . . . . . . . . . . . 17

\begin{tabular}{|llr}
\hline 5 & Die Zukunft von OpenBib & 19
\end{tabular}

5.1 Teilhabe der Nutzer am OPAC . . . . . . . . . . . . . . . . . . . . . . . . . . . 19

5.2 Weitere Mashups . . . . . . . . . . . . . . . . . . . . . . . . . . . 19

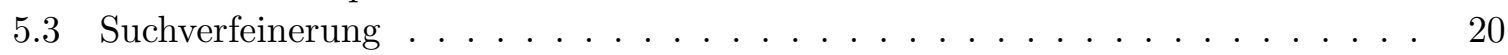

5.4 Weitere Recherche-Backends . . . . . . . . . . . . . . . . . . . . . . . . . . . . 20

$\begin{array}{lll}6 & \text { Fazit } & 20\end{array}$

\section{KUG und OpenBib}

Die Entwicklung des OpenBib Recherche-Portal $\mathrm{S}^{1}$ ist eng mit dem IGK-Projekt (InstitutsGesamtKatalog) verknüpft, das Anfang der 90er Jahre an der Universitäts- und Stadtbibliothek Köln (USB Köln) angesiedelt wurde und dessen Nachfolger KUG (Kölner UniversitätsGesamtkatalog $^{2}$ ), der 2002 an den Start ging. Vorrangiges Ziel der Projekte war die Schaffung eines universitätsweiten bibliothekarischen Gesamtkataloges, bestehend aus den Daten der USB, der Zentralbibliothek für Medizin (ZB MED) sowie der mehr als 140 Institute bzw. Seminare an der Universität zu Köln, und damit die Umsetzung der Vorgabe des Ministeriums für Wissenschaft und Forschung des Landes Nordrhein-Westfalen nach „funktionaler Einschichtigkeit

\footnotetext{
${ }^{1}$ OpenBib - Das OpenSource Recherche-Portal.

http://www.openbib.org/ (Zugriff am 30.3.2007)

${ }^{2}$ KUG - Kölner UniversitätsGesamtkatalog.

http://kug.ub.uni-koeln.de/ (Zugriff am 30.3.2007)
}

O. Flimm, Die Open-Source-Software OpenBib an der USB Köln - Überblick und Entwicklungen in Richtung OPAC 2.0 in: BIBLIOTHEK. Forschung und Praxis, Jg. 31 (2007) Nr. 2 
in zweischichtigen Bibliothekssystemen". Derzeit stellt das an der USB Köln eingesetzte KUG Recherche-Portal (kurz „KUG“, Abb. 1) den primären Einsatzort von OpenBib dar.

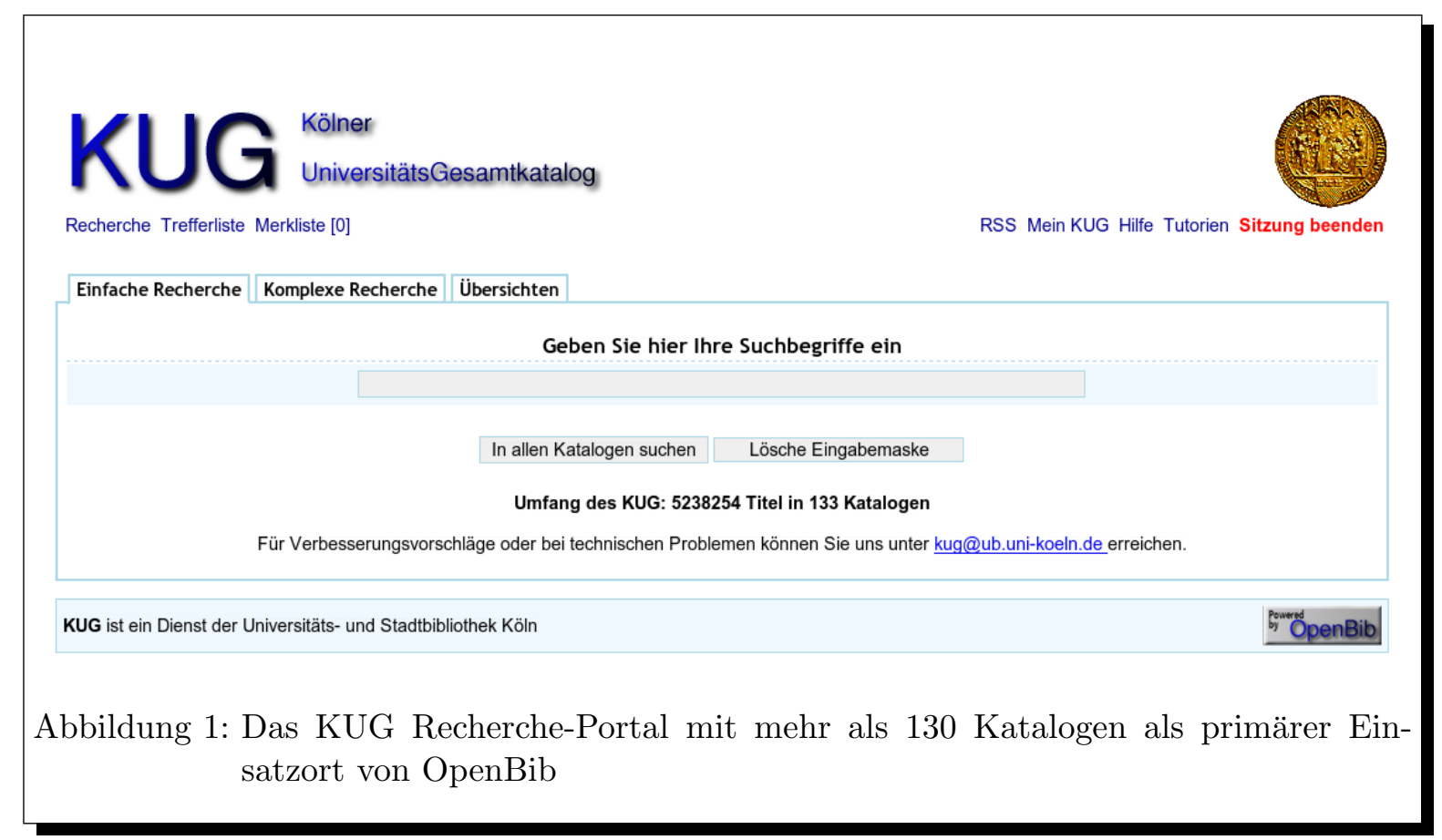

Eine grundlegende Anforderung war von Beginn an die Integration vieler einzelner Kataloge unter einer einheitlichen Suchoberfläche. Waren die Kataloge im Instituts- und Seminarbereich zunächst noch über verschiedene Bibliothekssysteme - u.a. BISLOK, Allegro, Lars, Lidos, FileMaker - verteilt, fand mit dem KUG-Projekt eine Konsolidierung auf das einheitliche integrierte Bibliothekssystem SISIS-SunRise der Firma OCLC PICA mit seinen Modulen Katalogisierung, Ausleihe und Erwerbung statt, das bereits bei der USB und der ZB MED im Einsatz war. Das System wird auf einem zentralen Datenbankserver betrieben, auf dem die Bestände eines jeden Instituts in eigenen Datenbanken gekapselt sind. Neben Katalogdaten aus SISIS-SunRise sind in das Portal weitere Katalog- und Datenquellen integriert - u.a. EconBiz und OAI-Repositories wie der Hochschulschriftenserver der Universität zu Köln.

Die Präsentation sehr vieler heterogener Daten und die sukzessiv gemachten Erfahrungen seit Anfang der 90er Jahre haben viele Design-Entscheidungen bei der Entwicklung von OpenBib maßgeblich beeinflusst. Es stehen nicht primär Funktionalitäten eines einzelnen Kataloges im Vordergrund, sondern vielmehr die Erschließung von Funktionalitäten für die Gesamtheit aller Kataloge und Datenbanken. Sehr oft ist damit ein Abwägungsprozess verbunden - wie z.B. bei der Anreicherung der Kataloge mit digitalisierten Inhaltsverzeichnissen (s.u.) - bei dem zugunsten eines maximalen Nutzens für alle Kataloge entschieden wird anstelle minimaler Vorteile für einen einzelnen Katalog. 


\subsection{Entkopplung von Portal und Bibliothekssystemen}

Ein Grundstein für die erfolgreiche Umsetzung der Anforderungen des KUG-Projektes mit OpenBib ist eine vollständige Entkopplung der Katalogdaten im Portal von den Ursprungssystemen. Die Voraussetzung dazu ist der Vollzugriff auf die Daten aller Kataloge, was im KUG-Projekt von Anfang an gegeben war.

Auf einem Portal-Server sind jeweils die bibliographischen Daten aller Kataloge in getrennten SQL-Datenbanken abgelegt - einem Katalog aus einem (theoretisch beliebigen) UrsprungsBibliothekssystem entspricht somit immer eine 'lokale' Datenbank auf allen Portal-Servern. Jeder Portal-Server umfasst also immer alle Katalogdaten und ist diesbezüglich autonom. Die nächtliche Synchronisation der Katalogdaten auf den Portal-Servern mit den Ursprungssystemen geschieht voll automatisiert. Darüber hinaus verfügt jede Datenbank über einen eigenständigen Suchmaschinen-Index, der alternativ für Rechercheanfragen oder Treffermengenanalysen herangezogen werden kann.

Eine direkte Kopplung des Recherche-Portals ist lediglich zur Ausleih-Komponente der jeweiligen Bibliothekssysteme notwendig - im Wesentlichen zur sekundengenauen Ermittlung des Ausleihstatus, zur Authentifizierung und zur Anzeige der Benutzerkonten. Diese Verbindung wird über SOAP-basierte WebServices realisiert.

Folgende Vorteile sind mit der Entkopplung der bibliographischen Daten von den Ursprungssystemen verbunden:

- Die Ursprungs-Bibliothekssysteme werden von Rechercheanfragen entlastet. Bei Wartungsarbeiten und Software-Upgrades der Bibliothekssysteme ist weiterhin eine Recherche über das Portal möglich.

- Durch eine „intelligente“ Lastverteilung zwischen verschiedenen Portal-Servern wird das Portal sehr skalierbar. Etwaige Performance-Engpässe, z.B. verursacht durch kontinuierlich steigende Rechercheanfragen, können sehr schnell durch Hinzunahme eines neuen Servers gelöst werden.

- Die Katalogdaten werden auf den Portal-Servern mit einer fest definierten Struktur in den Datenbanken abgelegt. Damit verfügt das Portal über eine einheitliche Datenbasis, auf deren Grundlage neue Dienste und Funktionen realisiert werden können, die in keinem der Usprungssysteme oder nur vereinzelt existieren.

Der Zugriff auf eine einheitliche Datenbasis ist eine zentrale Voraussetzung dafür, dass in einem Recherche-Portal wie dem KUG mit seinen mehr als 130 Datenbanken und über 5.2 Mio. Titeln in einem größeren Maßstab durch neue Funktionen ein Mehrwert für den Nutzer geschaffen wird.

Hier unterscheidet sich OpenBib mit seiner eigenen Datenhaltung grundsätzlich von herkömmlichen Recherche-Portalen, die Anfragen lediglich über standardisierte Protokolle wie Z39.50 an verschiedene Zielsysteme weiterleiten und die gelieferten Ergebnisse präsentieren. Bei diesen Portalen besteht eine sehr große Abhängigkeit von den zum Teil höchst unterschiedlichen Fähigkeiten der abgefragen entfernten Systeme, die damit eine wesentliche Begrenzung dar- 
stellen. OpenBib ist aber nicht das einzige Portal, das von einheitlich abgelegten Daten profitiert. Ein anderes Beispiel ist der innovative Dreiländerkatalog ${ }^{3}$ des hbz, der komplett auf Suchmaschinen-Technologie basiert und mit dem Konzept des Suchraumes ${ }^{4}$ auf einen Suchindex zugreift.

\subsection{Flexibilität durch Open-Source-Software}

Ein zweiter Grundstein für den Projekt-Erfolg ist eine größtmögliche Flexibilität bei der Implementierung neuer Funktionen. Zu deren Realisierung ist ein vollständiger Zugriff auf alle technischen Komponenten des Portals und deren Änderung bzw. Erweiterung zwingend notwendig.

Diese Anforderungen werden nur durch den Einsatz von Open-Source-Komponenten erfüllt, bei denen definitionsgemäß - im Gegensatz zu kommerzieller Standardsoftware - ein vollständiger Zugriff auf den Quell-Code möglich ist. Erst mit dieser vollständigen Kontrolle über die Software wird eine sehr schnelle Reaktion auf Trends und neue Technologien ermöglicht - dies im Übrigen auch noch kostengünstig.

Aufgrund dieser Flexibilität lassen sich Projekte sehr schnell umsetzen. Ein gutes Beispiel hierfür ist die „Digitale Einbandsammlung der USB Köln“, die von der ersten Konzeption bis zur finalen Umsetzung nur wenige Monate benötigt 5 .

Der Einsatz von Open-Source-Software am Beispiel von OpenBib hat sich auch im Produktionsbetrieb im Vergleich zu kommerzieller Standardsoftware als sehr vorteilhaft erwiesen:

- Erweiterungen werden umgehend selbständig vorgenommen und Probleme in der Regel deutlich schneller gelöst.

- Von unseren Benutzern an uns herangetragene Wünsche werden ebenfalls schneller umgesetzt.

- Release-Zyklen der Software werden selbst vorgegeben.

- Die Integration von anderen Software-Produkten über standardisierte Schnittstellen ist mit wenig Aufwand möglich.

\section{Ursprung und Technik von OpenBib}

Die Portalsoftware OpenBib ist seit ihrer ersten Version aus dem Jahr 1997, in der sie als „Freizeitprojekt" begonnen wurde und historisch noch unter dem Namen 'biblio' firmierte,

\footnotetext{
${ }^{3}$ Dreiländerkatalog.

http://suchen.hbz-nrw.de/ (Zugriff am 30.3.2007)

${ }^{4}$ hbz: Suchraum.

http://www.hbz-nrw.de/angebote/suchraum/ (Zugriff am 30.3.2007)

${ }^{5}$ Vgl. Regine Boeff, Oliver Flimm: Von der traditionellen zur digitalen Sammlung historischer und künstlerischer Bucheinbände der USB Köln mit einem Einblick in die technische Konzeption der Datenbank ; in: Bibliothek. Forschung und Praxis. Jahrgang 30 (2006) Nr. 1, S.63-68
} 
als Open-Source-Software verfügbar. Als Garant für die Freiheit ${ }^{6}$ dieser Software und gegen unerwünschte kommerzielle Vereinnahmung wurde bewusst die schon bei Linux verwendete GNU General Public License (GPL) gewählt. Schon wenig später - im Oktober 1997 - wurde biblio/OpenBib von der USB zur Realisierung des KVIK (Kölner Virtueller InstitutsgesamtKatalog7, dem Vorgänger des KUG) genutzt, mit zuletzt knapp 750.000 Titeln in 74 Katalogen im Juni 2002.

OpenBib basiert seinerseits wieder auf verbreiteten Open-Source-Komponenten - speziell Linux, Apache, MySQL und Perl. Damit handelt es sich um ein typisches LAMP-System mit einer niedrigen Einstiegsschwelle für einen erfahrenen LAMP-Entwickler.

Von ihrer ersten Version entwickelte sich die Software von einer reinen Sammlung an CGISkripten mit wenigen mod_perl-Elementen aus den Jahren 1997-2004 zu einer vollständig mod_perl-basierten, direkt in den Apache-Webserver integrierten Webanwendung. In dieser Webanwendung wird zusammen mit den zugrunde liegenden Datenbanken sowie einem Templating-System (Perl Template Toolkit) das sog. MVC-Design-Pattern realisiert und damit speziell die sinnvolle Trennung von Programm-Logik und Darstellung. Dadurch lassen sich auch sehr gut die entsprechenden Zuständigkeiten für Programmierung und Web-Design an verschiedene Mitarbeiter verteilen.

\subsection{Kaskadierung von Templates, CSS und Texten}

Durch die Verwendung der Templates können die Web-Oberflächen differenziert dargestellt und mit geringem Aufwand angepasst werden. Um den Aufwand für den Betrieb sehr vieler visuell eigenständiger Portal-Sichten zu minimieren, lassen sich die Templates katalog- und sichtspezifisch kaskadieren. Für eine einzelne Katalogsicht müssen damit nicht jeweils alle Templates für jede Sicht getrennt verwaltet, sondern lediglich die geänderten Templates an fest definierten Stellen abgelegt werden. Vor der Ausgabe eines Templates wird zunächst überprüft, ob es ein spezielles Template für diesen Katalog oder diese Sicht gibt - und dieses gegebenenfalls verwendet. Falls nicht, kommt das zugehörige Standard-Template zur Anwendung.

Wie bei den Templates lassen sich durch einen ähnlichen Mechanismus auch Cascading Stylesheets (CSS) und auszugebende Texte katalog- und sichtspezifisch kaskadieren. Die Möglichkeit, in Abhängigkeit vom Katalog andere Texte für identische Katalogkategorien zu vergeben, wird im KUG ausgiebig für Spezialkataloge mit alternativen Kategorienamen genutzt, wie der Einbandsammlung und der Portraitsammlung. So tritt bei der Portraitsammlung beispielsweise die Bezeichnung 'Maler, Zeichner' an die Stelle von 'Verfasser' in der MAB2-Kategorie 100.

Im KUG werden mit diesen einfachen Kaskadierungs-Techniken ausgehend von einfachen Institutssichten bis hin zu aufwändigeren Fach-Portalen insgesamt 144 unterschiedliche PortalVersionen im Rahmen nur einer OpenBib-Softwareinstallation angeboten.

\footnotetext{
${ }^{6}$ Free Software Foundation (FSF) / GNU Project: The Free Software Definition.

http://www.gnu.org/philosophy/free-sw.html (Zugriff am 30.3.2007)

${ }^{7}$ Kölner Virtueller InstitutsgesamtKatalog.

http://kvik.ub.uni-koeln.de/ (Zugriff am 30.3.2007)
} 


\subsection{Recherche-Backends, UTF8-Encoding und I18N}

Für die Suche im Portal stehen zwei alternative Recherche-Backends zur Verfügung. Neben einer klassischen Recherche über SQL kann auch ein Suchmaschinen-Backend verwendet werden. Dieses bietet weitergehende Möglichkeiten wie z.B. Treffermengen- sowie Termanalysen und basiert auf der Open-Source-Suchmaschinentechnologie von Xapian ${ }^{8}$.

Im KUG wird der Suchmaschinen-Index für die „Einfache Recherche“ und das SQL-Backend für die „Komplexe Recherche“ verwendet. Während das Suchmaschinen-Backend gerade bei alltäglichen Suchanfragen, wie sie von einem Großteil der Recherchierenden gestellt werden, seine Stärken auspielt, ist diese Technologie weniger für sehr spezialisierte Recherche-Anforderungen geeignet, wie sie z.B. aus dem bibliothekarischen Bereich unserer Nutzer gefordert werden. Deren Anforderungen können nur durch das SQL-Backend erfüllt werden.

Die Datenbankstruktur für die Katalogdaten ist sehr flexibel. MAB2 wird standardmäßig als Format sowohl in den SQL-Datenbanken wie auch für die vereinheitlichten Meta-Daten genutzt. Grundsätzlich sind aber auch andere Kategorienschemata oder Meta-Datenformate - wie z.B. MARC21 - mit entsprechendem Anpassungsaufwand möglich.

Als Standard-Encoding für das Portal wird zeitgemäß UTF8 verwendet. Damit lassen sich auch Katalog-Bestände aus UTF8-fähigen Bibliothekssystemen in das Portal integrieren.

Mit der Verwendung von GNU gettext kann das Portal grundsätzlich auch in alternativen Sprachversionen angeboten werden. Derzeit wird der KUG nur in deutscher Sprache angeboten. Eine Übersetzung in die englische Sprache wurde bereits begonnen.

\subsection{Administration und Betrieb}

Gerade die Administration von vielen Datenbanken in einem Portal ist ein sehr wichtiger - wenn auch für den Endanwender weitgehend unsichtbarer - Aspekt beim Betrieb des Portals. Aus diesem Grund verfügt OpenBib über eine bequeme, web-basierte Administrations-Oberfläche (Abb. 2), die den Arbeitsaufwand wesentlich minimiert. Neben den Katalogen lassen sich dort auch die Sichten, RSS-Feeds usw. konfigurieren sowie die aktiven Sessions überwachen.

Die Anforderungen von OpenBib an die Rechnerausstattung sind bescheiden. Das KUG Recherche-Portal wird lediglich mit drei Doppel-Pentium-III Servern (1.16 GHz CPU, 4 GB RAM) im RAID-Level 1 aus dem Jahr 2002 betrieben. Mit dieser Ausstattung konnten im Jahr 2006 insgesamt 557.445 Benutzer-Sessions mit mehr als 4 Mio. Recherche-Anfragen problemlos bewältigt werden, davon knapp 400.000 Anfragen über die Einbindung des KUG in die Digitale Bibliothek NRW (DigiBib9 s.u.).

Die Infrastruktur für das OpenBib-Projekt ${ }^{10}$ wird von BerliOs 41 bereitgestellt. Dort findet

\footnotetext{
${ }^{8}$ The Xapian Project.

http://www .xapian.org/ (Zugriff am 30.3.2007)

${ }^{9}$ Digitale Bibliothek (DigiBib).

http://www.digibib.net/ (Zugriff am 30.3.2007)

${ }^{10}$ BerliOS Developer: Project Info - OpenBib Recherche-Portal.

http://developer.berlios.de/projects/openbib/ (Zugriff am 30.3.2007)

${ }^{11}$ BerliOS - The Open Source Mediator.
} 
auch die ausschließliche Entwicklung über ein CVS-Repository ${ }^{12}$ statt.

\section{KUG \\ UniversitätsGesamtkatalog}

\begin{tabular}{|l|l|l|}
\hline Kataloge Views Sessions Logout \\
\hline
\end{tabular}

Neuen Katalog anlegen

Fakultät

Fakultätsungebunden

System DB-Name Sigel URL

Bereits existierende Kataloge

Fakultät

RSS Beschreibung

Fakultätsungebunden @ USB Köln

Fakultätsungebunden

(\$) Studentenbüchere

ه Universitätsarchiv

akultätsungebunden

Zentrum für Angewandte Informatik

Fakultätsungebunden

(RRZK)

Fakultätsungebunden

(1) Gleichstellungsbeauftragte

Staatliches Studienkolleg für

Fakultätsungebunden

Ausländische Studierende

Fakultätsungebunden

(1talienisches Kulturinstitut

Fakultätsungebunden

ه Zeitschriften der Institute

Fakultätsungebunden

Fakultätsungebunden

Wirtschafts- u.

Sozialwissenschaftliche Fakultät

Wirtschafts- $u$.

Sozialwissenschaftliche Fakultät

Wirtschafts- $u$.

Sozialwissenschaftliche Fakultät

ه Verschollene Werke

Deutsche Zentralbibliothek für

Medizin (ZB MED)

ه Wirtschaftsarchiv der WiSo-Fakultät

Seminar für Finanzwissenschaft

Finanzwissenschaftliches Forschungsinstitut

Z39.50:-

Abbildung 2: Die Administrations-Oberfläche des Portals

\section{Einsatz in Fach-Portalen}

Durch die flexiblen Möglichkeiten der Anpassung über eigene Sichten war die Software des Recherche-Portals predestiniert für den Einsatz in weiteren Projekten.

Hier sind insbesondere die folgenden eigenständigen Fach-Portale zu nennen:

- Die Digitale Einbandsammlung der USB Köln ${ }^{13}$

- Die Virtuelle Bibliothek Elise und Helene Richter ${ }^{14}$ als Teil der NS-Provenienzforschung in der USB Köln

http://www.berlios.de/ (Zugriff am 30.3.2007)

${ }^{12}$ OpenBib CVS-Repository bei BerliOS.

http://cvs.berlios.de/cgi-bin/viewcvs.cgi/openbib/openbib/ (Zugriff am 30.3.2007)

${ }^{13}$ Digitale Einbandsammlung der Universitäts- und Stadtbibliothek Köln.

http://einbandsammlung.ub.uni-koeln.de/ (Zugriff am 30.3.2007)

${ }^{14}$ Virtuelle Bibliothek Elise und Helene Richter.

http://richterbibliothek.ub.uni-koeln.de/ (Zugriff am 30.7.2007)

O. Flimm, Die Open-Source-Software OpenBib an der USB Köln - Überblick und Entwicklungen in Richtung OPAC 2.0 in: BIBLIOTHEK. Forschung und Praxis, Jg. 31 (2007) Nr. 2 
- Die Virtuelle Bibliothek Historische Bestände im Rheinland 15 (Abb. 3 )

- Die Portraitsammlung der USB Köln[16

- Europäische Städte- und Landschaftsbilder des 16. und 17. Jahrhundert:\$17

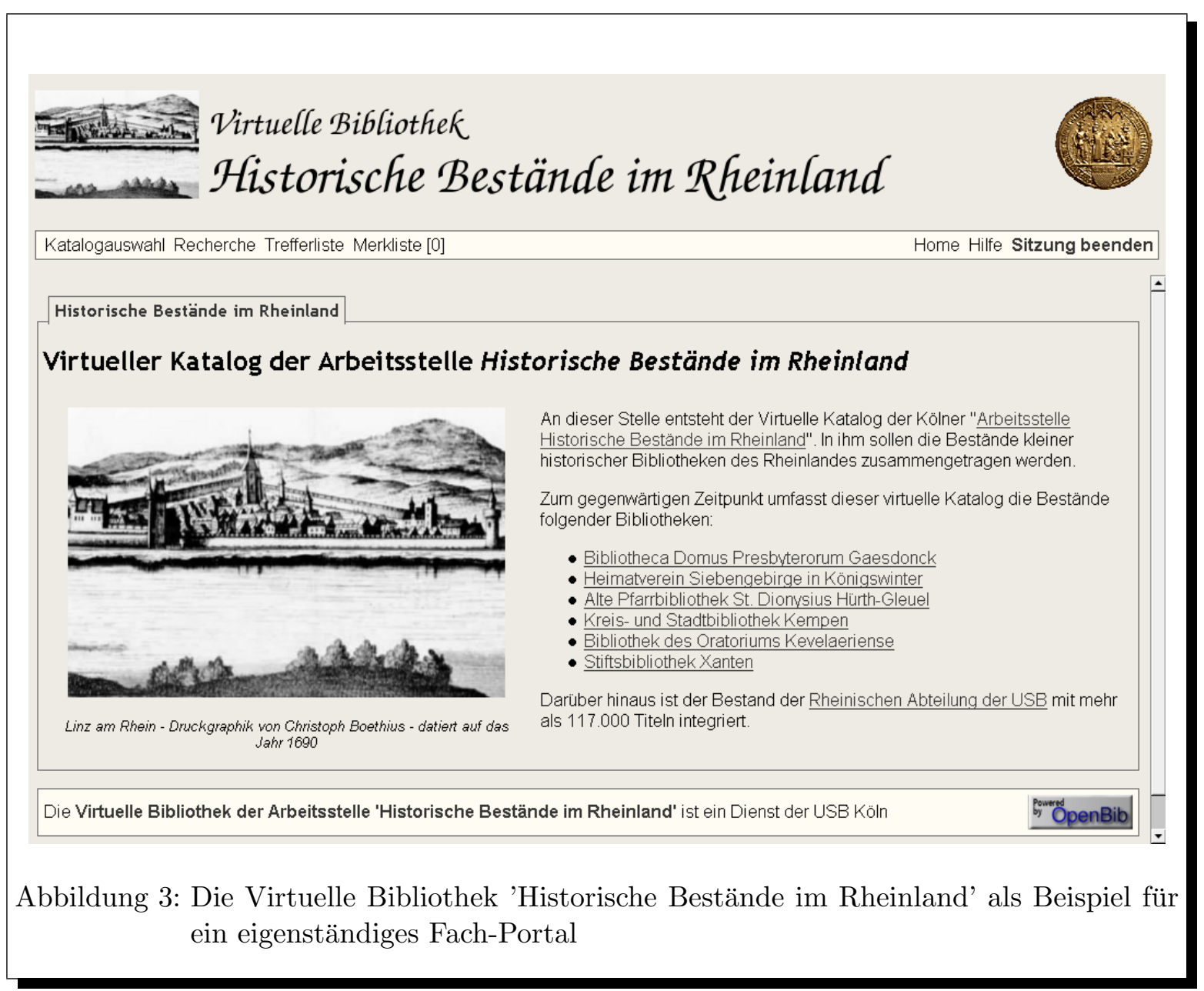

\section{Anreicherungen, Mashups und OPAC 2.0}

Seitdem Michael Casey im Jahr 2005 den Begriff „Library 2.0“ in Anlehnung an das „Web 2.0" erstmals erwähnte, wurde verstärkt nach nutzbringenden Anwendungen von Techniken und Prinzipien des Web 2.0 im Kontext einer Bibliothek geforscht. Ebenso wird geänderten Nutzungsgewohnheiten Rechnung getragen, die sich bei den Nutzern inzwischen durch das

\footnotetext{
${ }^{15}$ Virtuelle Bibliothek Historische Bestände im Rheinland. http://rheinlandbib.ub.uni-koeln.de/ (Zugriff am 30.3.2007)

${ }^{16}$ Portraitsammlung der Universitäts- und Stadtbibliothek Köln. http://portraitsammlung.ub.uni-koeln.de/ (Zugriff am 30.3.2007)

${ }^{17}$ Europäische Städte- und Landschaftsdarstellungen des 16. und 17. Jahrhunderts. http://landschaftsbilder.ub.uni-koeln.de/ (Zugriff am 30.3.2007)
} 
Internet und den dort angesiedelten Diensten (u.a. Amazon und Google) manifestiert haben. Bei allen Überlegungen werden aktuelle Anforderungen eines Bibliotheksbenutzers an eine zeitgemäße Bibliothek und ein Mehrwert für ihn in den Mittelpunkt gestellt. Der OPAC als eine zentrale Bibliotheksanwendung für den Nutzer rückt im Zuge dieser Verbesserungen als "OPAC 2.0" in den Vordergrund.

Wie andere Kataloge auch, z.B. der innovative XOPAC ${ }^{18}$ aus Karlsruhe, hat der KUG mit OpenBib viele Ideen aufgegriffen und bereits umgesetzt. Speziell die Idee des „sozialen Web“ ist in einige der aktuellen Entwicklungen eingeflossen.

Die Erweiterungen von OpenBib am Beispiel des KUG lassen sich in verschiedene Bereiche einordnen:

- Grundsätzliche Anreicherung der Katalogdaten mit weiteren Informationen

- Digitalisierte Inhaltsverzeichnisse

- Funktionen, die dem Nutzer einen Überblick über Kataloginhalt und -nutzung und damit grobe Orientierungshilfen bieten

- Tag-Clouds für im Katalog vergebene Schlagworte, Notationen, Körperschaften, Personen sowie durch Nutzer vergebene Tags

- Popularitäts-Funktion: Top 20 Titel eines Kataloges bezogen auf Ausleihe und Nutzung im KUG

- Funktionen, die aufgrund der Katalogstruktur oder der Analyse des Nutzungsverhaltens direkte Hilfestellungen in Treffermengen und Einzeltrefferansichten anbieten. Das sind u.a.:

- Recommender-Funktion: „Das könnte Sie interessieren“

- Popularitäts-Funktion: Sortierung nach Popularität

- Individuelles und gemeinschaftliches Indexieren („Tagging“)

- Tag-Clouds für die Verteilung von relevanten Termen in Treffermengen

- Drilldowns in Treffermengen

- Funktionen, die dem Nutzer über Zugriffsschnittstellen oder Mashups weitere Nutzungsmöglichkeiten bieten

- RSS-Feeds für Neuzugänge in den Katalogen

- Mashup mit der Social-Software BibSonomy

- Mashup mit der Wikipedia für Personen und ISBN-Suche

- Mashup mit weiteren Recherche-Portalen

Im Folgenden werden einige dieser nutzerzentrierten Funktionen von OpenBib im KUG näher ausgeführt.

${ }^{18} \mathrm{X}$-OPAC Extendable Online Public Access Catalog.

http://www.xopac.org/ (Zugriff am 30.3.2007)

O. Flimm, Die Open-Source-Software OpenBib an der USB Köln - Überblick und Entwicklungen in Richtung OPAC 2.0 in: BIBLIOTHEK. Forschung und Praxis, Jg. 31 (2007) Nr. 2 


\subsection{Ergebnisanreicherung aller KUG-Datenbanken durch gescannte Inhaltsverzeichnisse}

Unter der Federführung des Hochschulbibliothekszentrums NRW (hbz), unterstützt vom Ministerium für Innovation, Wissenschaft, Forschung und Technologie des Landes NordrheinWestfalen und durchgeführt von der Firma ImageWare Components GmbH in Zusammenarbeit mit den beteiligten Bibliotheken, wurden seit Herbst 2005 an der USB Köln und der ZB MED im Projekt 180T Inhaltsverzeichnisse von Büchern gescannt und mit einer OCRSchrifterkennung bearbeitet (Wirtschafts- u. Sozialwissenschaften, Medizin). Ziel ist das sog. Catalogue Enrichment 19 , also sowohl eine Such- wie auch Ergebnisanreicherung in OnlineKatalogen.

Da dieses Projekt in seiner eigentlichen Konzeption auf die Anreicherung des USB- bzw. Verbundkataloges ausgerichtet ist, wurde von uns dieses Konzept lokal für einen Einsatz im Institutsumfeld in der Form eines allgemeinen Anreicherungskonzeptes für den KUG erweitert, so dass wir die in diesem Projekt gewonnenen digitalen Inhaltsverzeichnisse der USB auch für alle Institute und Seminare nutzbar machen können.

Über eine zentrale Anreicherungsdatenbank können zusätzliche Inhalte katalogübergreifend in die vorhandenen Katalogdaten eingeblendet oder für eine Suche herangezogen werden. Grundlage ist eine vorhandene ISBN.

Diese Einschränkung ist notwendig, wenn ein Nutzen für eine größtmögliche Anzahl an Katalogen erzielt werden soll. Denn Anreicherungsinformationen sind nun nicht mehr mit einem speziellen Titel in einem speziellen Katalog verknüpft, sondern ganz allgemein - und damit automatisch nutzbar für alle Kataloge - über die ISBN. Der jeweilige Titel in irgendeinem Katalog „weiß“ von einer möglichen Anreicherung nichts. Erst bei der Einzeltrefferanzeige werden für einen konkreten Titel Katalog- und Anreicherungsdaten kombiniert und dann ausgegeben.

Bei der Inhaltsanreicherung durch digitalisierte Inhaltsverzeichnisse bedeutet diese "portalzentrierte Sichtweise“ im Vergleich zur einer „einzelkatalogzentrierten Sichtweise“ im konkreten Fall einen „hypothetischen Verlust" von knapp 9 Prozent an Inhaltsverzeichnissen für den USB Katalog zugunsten aller anderen. Dieser Titel-Anteil verfügt über keine ISBN.

Da in den USB Katalog jedoch die Inhaltsverzeichnisse ohnehin über regelmäßige MAB2Exporte aus dem Verbundkatalog Einzug finden, sind auch im USB-Katalog im Kontext des KUG schließlich wieder alle Inhaltsverzeichnisse zugreifbar.

Wie schon ausgeführt ist die Konzeption der Ergebnisanreicherung in OpenBib so allgemein gehalten, dass sie sich neben der Ergebnisanreicherung mit Inhaltsverzeichnissen auch mit anderen Zusatzinformationen nutzen lässt. So können beliebige Informationen, wie Nutzer-Reviews, Abstracts, Stichwortverzeichnisse (nach Absprache mit den entsprechenden Rechteinhabern) u.ä., dort zentral abgelegt werden und sind für alle Kataloge des OpenBib-Portals automatisch nutzbar.

${ }^{19}$ hbz: Catalogue Enrichment.

http://www.hbz-nrw.de/angebote/catalogue_enrichment/ (Zugriff am 30.3.2007)

O. Flimm, Die Open-Source-Software OpenBib an der USB Köln - Überblick und Entwicklungen in Richtung OPAC 2.0 in: BIBLIOTHEK. Forschung und Praxis, Jg. 31 (2007) Nr. 2 


\subsection{Individuelles und gemeinschaftliches Indexieren („Tagging“)}

Die Indexierung von Dokumenten durch vom Nutzer selbst gewählte „Etiketten“ oder MiniSchlagworte (engl. tags) hat sich im Bereich sozialer Software - z.B. in Web 2.0-Diensten wie BibSonomy, flickr oder del.icio.us - als ein effektiver Mechanismus bewährt, mit dem sich

1. Dokumente individuell organisieren und in Gruppen zusammenfassen lassen

2. diese zunächst individuell motivierten Tag-Sammlungen und Dokument-Gruppen zum Vorteil anderer Nutzer verwenden lassen

Dieser Mechanismus ist auch auf die Kennzeichnung von Titeln in Bibliothekskatalogen durch Nutzer übertragbar und deckt dort verschiedene Bereiche ab: Als „individuell strukturierbare Merkliste", als Alternative zur formalen und inhaltlichen Sacherschließung durch Bibliotheka$\mathrm{re}^{20}$ sowie als Instrument, um Nutzer auf weitere thematisch ähnliche Titel aufmerksam zu machen.

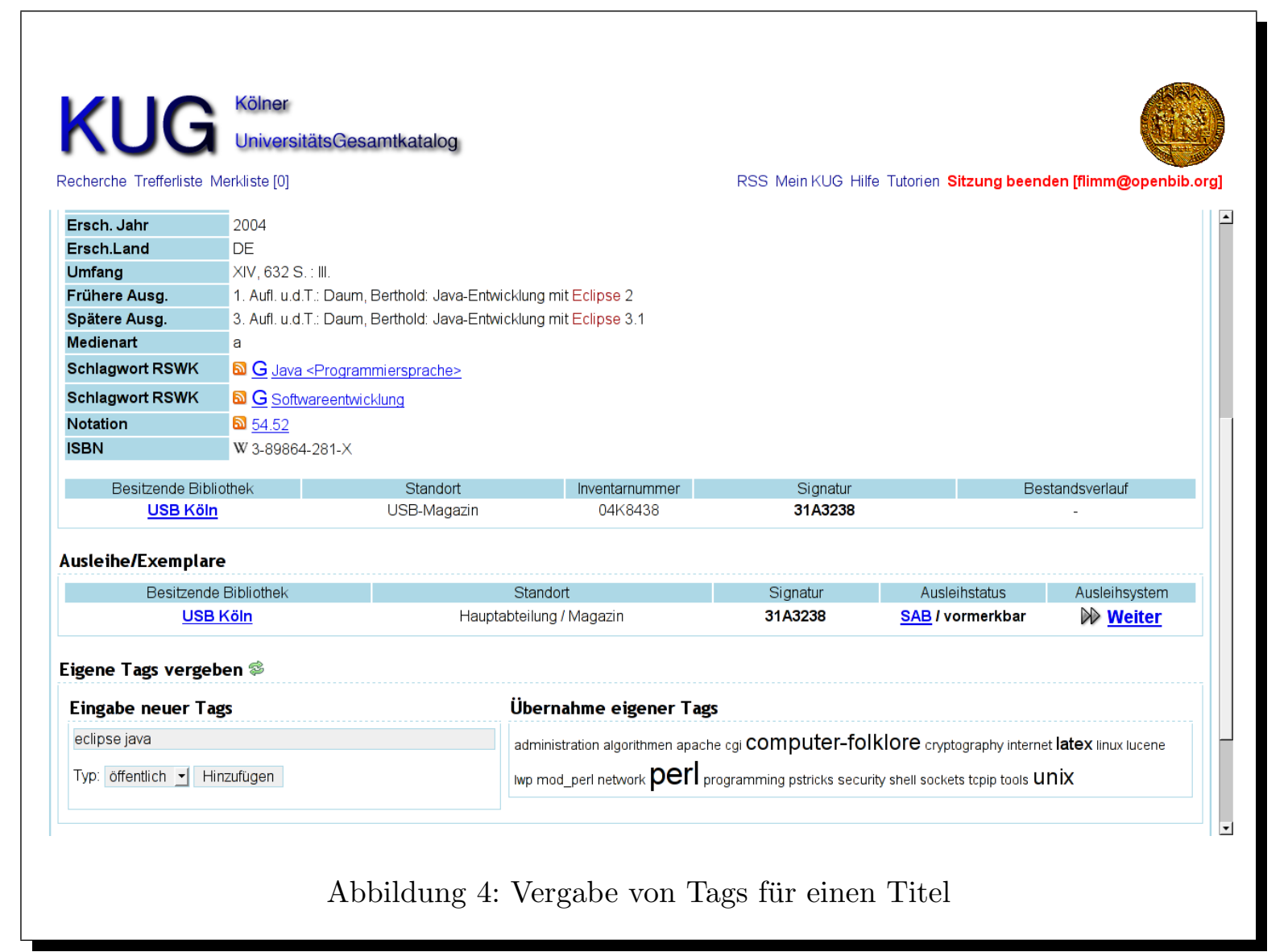

${ }^{20}$ Dies gilt insbesondere dann, wenn keine Sacherschließung mit Schlagworten oder Systematiken durch Bibliothekare vorgenommen wird, wie es z.B. bei einigen Katalogen im KUG der Fall ist. 
Von OpenBib wird sowohl individuelles wie auch gemeinschaftliches Indexieren ${ }^{21}$ unterstützt. Die Vergabe von Tags ist auf am Portal angemeldete Nutzer beschränkt.

Der bibliothekspolitischen Dimension als Alternative zu der von Bibliothekaren vorgenommenen Sacherschließung - „Soll der Nutzer überhaupt zum Katalog durch eigene Verschlagwortung beitragen dürfen? " - wird in OpenBib dadurch Rechnung getragen, dass zentral festgelegt werden kann, ob lediglich individuelles Indexieren in Form von „individuell strukturierbaren Merklisten" oder aber das gesamte Spektrum des gemeinschaftlichen Indexierens und Nutzens - also wirkliches „Social“-Tagging - freigegeben werden soll. Letzteres ist die Standardeinstellung in OpenBib.

Ein Problem, das beim gemeinschaftlichen, aber auch individuellen Indexieren auftreten kann, ist die Zersplitterung des "Tag-Raumes" durch die Verwendung von Umlauten, Groß-Kleinschreibung und verschiedenen Wortformen. Aus diesem Grund ist es sehr sinnvoll, dieser Zersplitterung sowohl durch eine aufgezwungene wie auch freiwillige Vereinheitlichung von Tags entgegen zu wirken.

OpenBib löst daher bei der Vergabe von Tags zu einem Titel automatisch Umlaute auf, transformiert in Kleinschreibung und eliminiert unerwünschte Zeichen in den Tags. Neben diesen aufgezwungenen Maßnahmen werden dem Nutzer zusätzlich als Orientierungshilfe für die TagWahl alle von ihm bereits generell vergebenen Tags sowie die von anderen Nutzern für den konkreten Titel bereits genutzten Tags in Form von Wortwolken (s.u.) dargestellt. Durch Klick auf einen Tag aus den Wortwolken wird dieser automatisch in das Eingabefeld eingetragen. Ferner kann für die Tags eines Titels festgelegt werden, ob diese nur privat oder auch öffentlich zugänglich sind.

Beim Aufruf eines einzelnen Titels werden alle als öffentlich definierten Tags zu diesem Titel ausgegeben. Über diese kann jeder Nutzer auf alle anderen so gekennzeichneten Titel zugreifen. Ist ein Nutzer am Portal angemeldet, dann werden zusätzlich seine eigenen Tags dargestellt und ihm darüber der Zugriff auf alle durch ihn damit verknüpften Titel ermöglicht.

Jenseits der Tags eines einzelnen Titels runden allgemeine Übersichten eines angemeldeten Nutzers über alle seine Tags und damit Titel sowie der von allen Nutzern für die jeweiligen Kataloge vergebenen Tags die Tagging-Funktionen in OpenBib ab.

\subsection{Tag-Clouds als Orientierungshilfe}

Zur Visualisierung von nutzerdefinierten Tags werden diese in vielen Web 2.0-Diensten als verlinkte „Wortwolken 22" (engl. tag cloud) dargestellt. Mit Hilfe dieser Wolken-Ansicht bekommt der Nutzer eine sehr gute Übersicht der vergebenen Tags sowie über deren Häufigkeit. Je öfter ein Tag verwendet wird, desto größer wird es dargestellt.

\footnotetext{
${ }^{21}$ Artikel Gemeinschaftliches Indexieren aus der Wikipedia. http://de.wikipedia.org/wiki/Gemeinschaftliches_Indexieren (Zugriff am 10.4.2007)

${ }^{22}$ Artikel Wortwolke aus der Wikipedia. http://de.wikipedia.org/wiki/Wortwolke (Zugriff am 10.4.2007)
} 


\section{KUG

EconBiz - Virtuelle Fachbibliothek Wirtschaftswissenschaften

Wolken Top 20

\begin{tabular}{l|l|l|l|l|l} 
Schlagworte & Systematiken/Notationen & Körperschaften/Institutionen & Personen & Nutzer-Tags
\end{tabular}

\section{Wolke der Schlagworte}

Aktienmarkt Arbeitgeberverband Arbeitsbeziehungen Arbeitsmarkt Arbeitsmarktpolitik Arbeitsorganisation Austria Bank Bankbetriebslehre Berufsverband Betriebliche Fortbildung

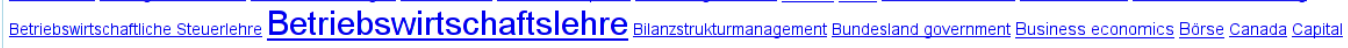

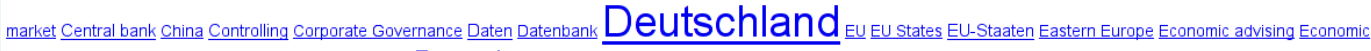
policy Economic research Economic research institute Economics Economists Electronic Commerce Empirische Forschung Energiepolitik Entwicklung Entwicklungshilfe Entwicklungsländer Entwicklungspolitik Erhebung Europa Europe European integration Europäische Union Fachhochschule Finanzierung Finanzierungstheorie Finanzmarkt Finanzpolitik

Finanzsektor Finanzwirtschaft Finanzwissenschaft Fliegen Flughafen Flugpassagier Forschung Forschungsinstitut Geldpolltik Germany Gesundheitspolltik Gesundheitswesen Gewerkschaft Gewerkschaftsorganisation Gewerkschaftspolitik Globalisierung Great Britain Großbritannien Handel Higher education Hochschule Informationsdienstleistung Informationsmanagement Informationssystem Informationstechnik Innovation Innovationsmanagement Interessenvertretung Interest group International economic relations Internationale Wirtschaftsbeziehungen Internet Investition Japan Kanada Kapitalmarkt Klein- und Mittelbetrieb Kommunikation Kooperation Krankenversicherung Kreditrisiko Landespolitik Landesregierung Leasing Lebensversicherung Logistik Lufftahrt Lufffracht Luftpost Luftverkehr Management Marketing Ministerium Mitarbeiter Nachnaltige Entwicklung Netzwerk Operations Research Organisation Organisationstheorie Organisatorisches Lernen Osteuropa Personalentwicklung Personalwesen Politikberatung Politikwissenschaft Porttolio Selection Risikomanagement Rückversicherung School of Economics $\underline{\text { School of economics } \underline{S c h w e i z}} \underline{\underline{S e c u r i t i e s ~ e x c h a n g e ~}} \underline{\text { Sozialpolitik }} \underline{\text { Sozialwissenschaft }} \underline{\text { Statistical authority }} \underline{\underline{\text { Statistical publication }}}$ Statistik Statistisches Amt Strategie Strategisches Management Sustainable development Switzerland Tarifpolitik USA Umweltpolitik Umweltschut $\underline{\text { United States University of }}$ applied sciences Unternehmen Unternehmensbewertung Unternehmensgründung Unternehmerverband Vereinigte Staaten verkehr Verkehrspolitik versicherung

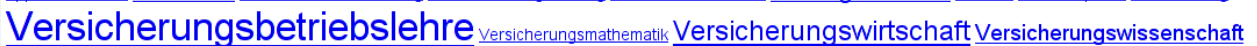
Verwaltungsreform Volkswirtschaft Volkswirtschaftslehre Weiterbildung Welt Wertpapierbörse Wettbewerb Wirtschaft Wirtschaftsforschung Wirtschaftsforschungsinstitut

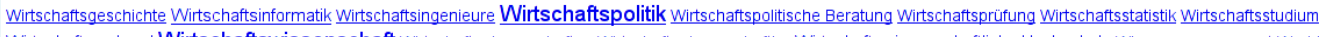
Wirtschaftsverband Wirtschaftswissenschaft Wirtschaftswissenschaften Wirtschaftswissenschaftler Wirtschaftswissenschaftliche Hochschule Wissensmanagement World Zentralbank economic policy economics environmental policy financial sector international organization ministry policy of a Bundesland social policy social science stock exchange sustainable development $\underline{\text { Osterreich }}$

KUG ist ein Dienst der Universitäts- und Stadtbibliothek Köln

Abbildung 5: Der Bestand von EconBiz als Beispiel für eine visuelle Aufschlüsselung nach Schlagworten mit Tag-Clouds

Gerade in einem Bibliothekskatalog ist die vorhande Verschlagwortung für den Nutzer oft weder durchschaubar noch direkt greifbar. Ob, wie und in welchem Ausmaß in einem Katalog verschlagwortet wurde, erschließt sich ihm vielfach nicht.

Hier bieten Wortwolken - auch an anderen Stellen des Kataloges jenseits der Schlagworte eine elegante Möglichkeit, um dem Nutzer „mehr Ein- und Übersicht“ zu bieten. In OpenBib werden solche „Übersichts-Wolken“ derzeit an mehreren Stellen eingesetzt.

1. bei der Verteilung der Schlagworte eines Kataloges, bezogen auf die $n$ häufigsten Schlagworte (Standard: $n=200$, Abb. 5). Ebenso werden auch die anderen Normdaten-Arten, wie Systematik, Körperschaften und Personen in Form einer Wortwolke für die einzelnen Kataloge dargestellt

2. bei der Verteilung der mit Tags individuell oder gemeinschaftlich indexierten Titel (titel-, nutzer- und katalog-spezifisch)

3. bei der Verteilung der relevanten Terme, bezogen auf deren Häufigkeit in einem ErgebnisTrefferset und bezogen auf die ersten $n$ Treffer (Standard: $n=200$ ) 
Weitere Einsatzgebiete sind möglich, wie z.B. der Verteilung der im Katalog von Nutzern verwendeten Suchbegriffe (Suchwolke). Suchwolken werden u.a. bereits im kommerziellen Findus Internet-OPAC ${ }^{23}$ verwendet, der vorrangig bei Stadt- u. Gemeindebüchereien eingesetzt wird 24 .

\subsection{Auswertung des Nutzungsverhaltens}

Dienste wie Amazon machen vor, wie eine Analyse des Nutzungsverhaltens in der OnlinePlattform zu einem Mehrwert für den Kunden führen kann. Entsprechend der zugrunde liegenden Datenbasis aus Verkäufen und der Auswahl konkreter Produkte für eine Detail-Ansicht, werden für den Kunden Funktionen wie „Kunden, die diesen Artikel kauften oder ansahen, haben auch... “ oder „Die beliebtesten Artikel“ (bezogen auf eine Recherche) realisiert.

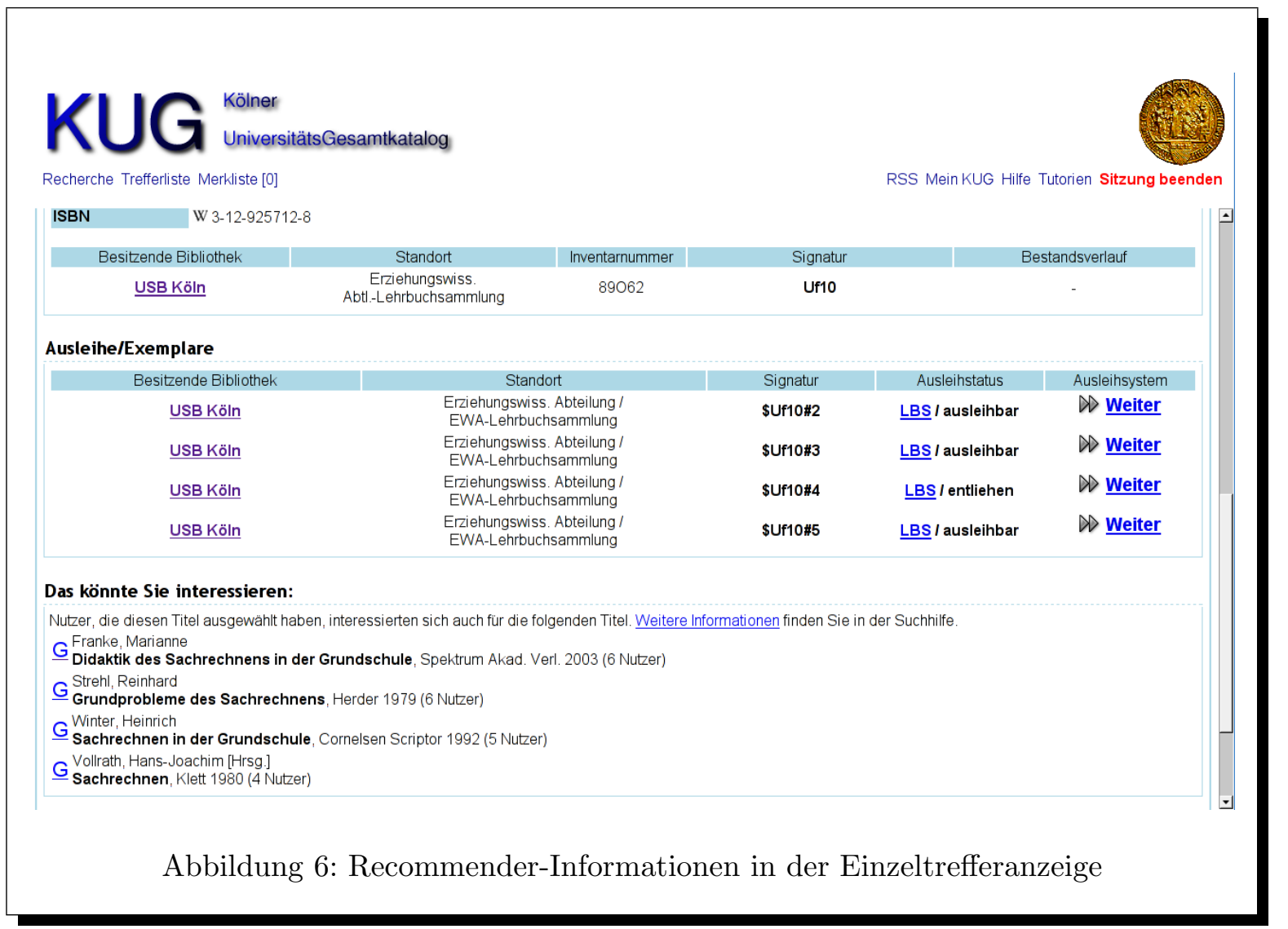

Dieses Potential steckt auch in Bibliothekskatalogen und entsprechende Funktionen können hier sinnvoll angeboten werden, wie z.B. der Karlsruher XOPAC zeigt.

Auch OpenBib bietet solche Funktionen für den KUG an. Dazu werden derzeit die Ausleihbewegungen sowie der Aufruf der Vollanzeige für Titel herangezogen. Diesen Informationen

\footnotetext{
${ }^{23}$ Findus Internet-OPAC. http://www.findus-internet-opac.de/ (Zugriff am 30.3.2007)

${ }^{24}$ Z.B. Bibliothek Wasserburg - Katalog (OPAC). http://www. wasserburg.de/de/bibliothek/katalog/, „Was andere Suchen“ (Zugriff am 1.3.2007)
} 
werden dann entsprechende anonymisierte Nutzer- bzw. Session-ID's zugeordnet.

Auf Grundlage der so gewonnenen Datenbasis werden im KUG folgende Funktionen angeboten:

Recommender-Funktion $\mathrm{Zu}$ einem konkreten Titel können andere Titel entsprechend des allgemeinen Nutzungsverhaltens unter der Überschrift „Das könnte Sie interessieren“ (Abb. 6) korreliert werden. Dazu werden nutzungsspezifische Überdeckungsprofile all jener anderen Titel erstellt, die Nutzer im Kontext des konkreten Titels angesehen bzw. ausgeliehen haben.

Popularitäts-Informationen in Trefferlisten Nach einer Recherche werden in der Trefferliste die absoluten Zugriffe anderer Nutzer auf die jeweiligen Titel ausgegeben. Zusätzlich kann der Nutzer pro Katalog bzw. katalogübergreifend nach diesen Popularitätsinformationen sortieren und so das Nutzungsverhalten anderer optional in die eigene RechercheStrategie einbeziehen.

Allgemeine Popularitäts-Informationen Für jeden Katalog können weitere allgemeine Informationen angeboten werden, wie z.B. die 20 meistgenutzten Titel usw.

\subsection{Drilldowns in Treffermengen}

Zur Suchverfeinerung in Treffermengen stellt OpenBib katalogweise optional neben Tag-Clouds für die relevantesten Terme auch - nach Kategorien aufgeschlüsselt - die relevantesten Kategorieinhalte dar. Ausgehend von diesen Inhalten kann die Treffermenge über Drilldowns weiter eingegrenzt werden. Drilldowns sind ein weit verbreitetes Mittel, um große und unübersichtliche Treffermengen für den Nutzer zu erschließen. OpenBib setzt diese Art von Drilldowns über Kategorieinhalte standardmäßig daher erst bei größeren Treffermengen ein, die aus mehr als 50 Titeln bestehen.

\subsection{RSS-Feeds}

Um den Nutzer über Neuzugänge in einem Katalog zu informieren, wurde bewusst eine Entscheidung gegen die Implementierung herkömmlicher E-mail-basierter Alerting-Dienste und stattdessen für den Einsatz der sehr viel flexibleren XML-basierten RSS-Technologie getroffen. RSS-Feeds bieten den Nutzern durch die geschickte Verwaltung über spezialisierte Programme deutlich mehr Nutzungsmöglichkeiten als Mails oder statische Webseiten So können solche Programme sich um die Sichtung der Daten kümmern, schon aufgerufene Titel von den noch nicht aufgerufenen farblich trennen, Informationen archivieren, Data-Mining in Verbindung mit spezialisierter Suchtechnologie einsetzen usw. Mit dieser Technik werden seit April 2006 Neuzugangslisten der Kataloge im KUG-Kontext angeboten. Das umfasst z.B. die letzten 50 generell in einen Katalog aufgenommenen Titel. Ebenso lassen sich die letzten 50 Titel auch bezogen auf einen konkreten Verfasser, eine Körperschaft, ein Schlagwort oder eine Notation als Feed ausgehend von einer Einzeltrefferanzeige abonnieren.

Zur Zeit werden im KUG für 113 Kataloge RSS-Feeds (Abb. 7) angeboten. Damit nimmt die USB Köln bei dem Einsatz dieser innovativen Technologie unter den großen wissenschaftlichen Bibliotheken deutschlandweit eine Vorreiterrolle ein. 


\section{KUG

ه Letzte 50 Neuaufnahmen (http://kug.ub. uni-koeln.de/portal/connector/rss/neuzugang/inst007. rdf) Italienisches Kulturinstitut

ه Letzte 50 Neuaufnahmen (http://kug.ub.uni-koeln.de/portal/connector/rss/neuzugang/inst900.rdf) USB Köln

ه Letzte 50 Neuaufnahmen (http://kug.ub.uni-koeln.de/portal/connector/rss/neuzugang/inst001, rdf) Zeitschriften der Institute

ه Letzte 50 Neuaufnahmen (http://kug.ub.uni-koeln.de/portal/connector/rss/neuzugang/instzs. rdf)

Zentrum für Angewandte Informatik (RRZK)

ه Letzte 50 Neuaufnahmen (http://kug.ub. uni-koeln.de/portal/connector/rss/neuzugang/insto06.rdf)

\section{RSS-Feeds: Wirtschafts- u. Sozialwissenschaftliche Fakultät}

Energiewirtschaftliches Institut

ه Letzte 50 Neuaufnahmen (http://kug.ub.uni-koeln.de/portal/connector/rss/neuzugang/inst119.rdf)

\section{FBV Fachbibliothek Versicherungswissenschaft}

ه Letzte 50 Neuaufnahmen (http://kug.ub.uni-koeln.de/portal/connector/rss/neuzugang/inst123.rdf)

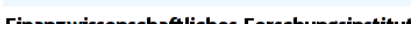

Abbildung 7: Auswahl aus insgesamt 113 RSS-Feeds für Neuzugänge in den jeweiligen Katalogen des KUG

\subsection{Mashups}

Durch die Kombination oder Integration bereits extern vorhandener Dienste - kurz Mashup ${ }^{25}$ genannt - lassen sich weitere sinnvolle Erweiterungen eines Bibliothekskataloges realisieren und dem Nutzer so einen Mehrwert bieten.

Im KUG werden mit OpenBib verschiedene Mashups zu externen Diensten angeboten:

BibSonomy In der Einzeltreffer-Anzeige (Abb. 8) wie auch in der Merkliste können die bibliographischen Angaben eines Titels direkt an BibSonomy ${ }^{26}$ gesendet werden. BibSonomy ist ein freier Social Bookmark Dienst ${ }^{27}$, der zudem auf die Verwaltung von BibliographieListen und deren Austausch/Verbreitung spezialisiert ist. Damit kann ein Nutzer direkt von dem Mehrwert profitieren, den Social-Software bietet und z.B. über entsprechende Tags weitere relevante Titel in BibSonomy finden.

${ }^{25}$ Artikel Mashup (Internet) aus der Wikipedia. http://de.wikipedia.org/wiki/Mashup_(Internet) (Zugriff am 30.3.2007)

${ }^{26}$ BibSonomy - A blue social bookmark and publication sharing system. http://www.bibsonomy.org/ (Zugriff am 30.3.2007)

${ }^{27}$ Entsprechend der Webseite genauer: Ein „social bookmark and publication sharing system“ (Zugriff am 30.3.2007) 
Wikipedia In der Einzeltreffer-Anzeige (Abb. 8) werden Personen sowie ISBNs direkt in die Wikipedia verlinkt. Wenn ein Nutzer Informationen zu einer Person oder einem Verfasser benötigt, kann er direkt eine Recherche nach der ensprechenden Person in der Wikipedia starten. Ebenso lässt sich bei vorhandener ISBN der ISBN-Suchservice der Wikipedia nutzen. Damit stehen dem Nutzer automatisch verschiedene Verbundkataloge, Buchhändler, Antiquariate usw. zur Verfügung, bei denen er direkt nach dem aktuell ausgewählten Titel recherchieren kann, ohne sich mit der jeweiligen Suchoberfläche auseinandersetzen zu müssen.

DigiBib, EZB, DBIS, MedPilot Ausgehend von einer jeden Recherche des Nutzers (und einer etwaigen Authentifizierung) im KUG kann mit Übernahme der Recherchebegriffe (und den Authentifizierungsinformationen im Falle der DigiBib) direkt in die Recherchefunktion der entsprechenden Portale gesprungen werden.

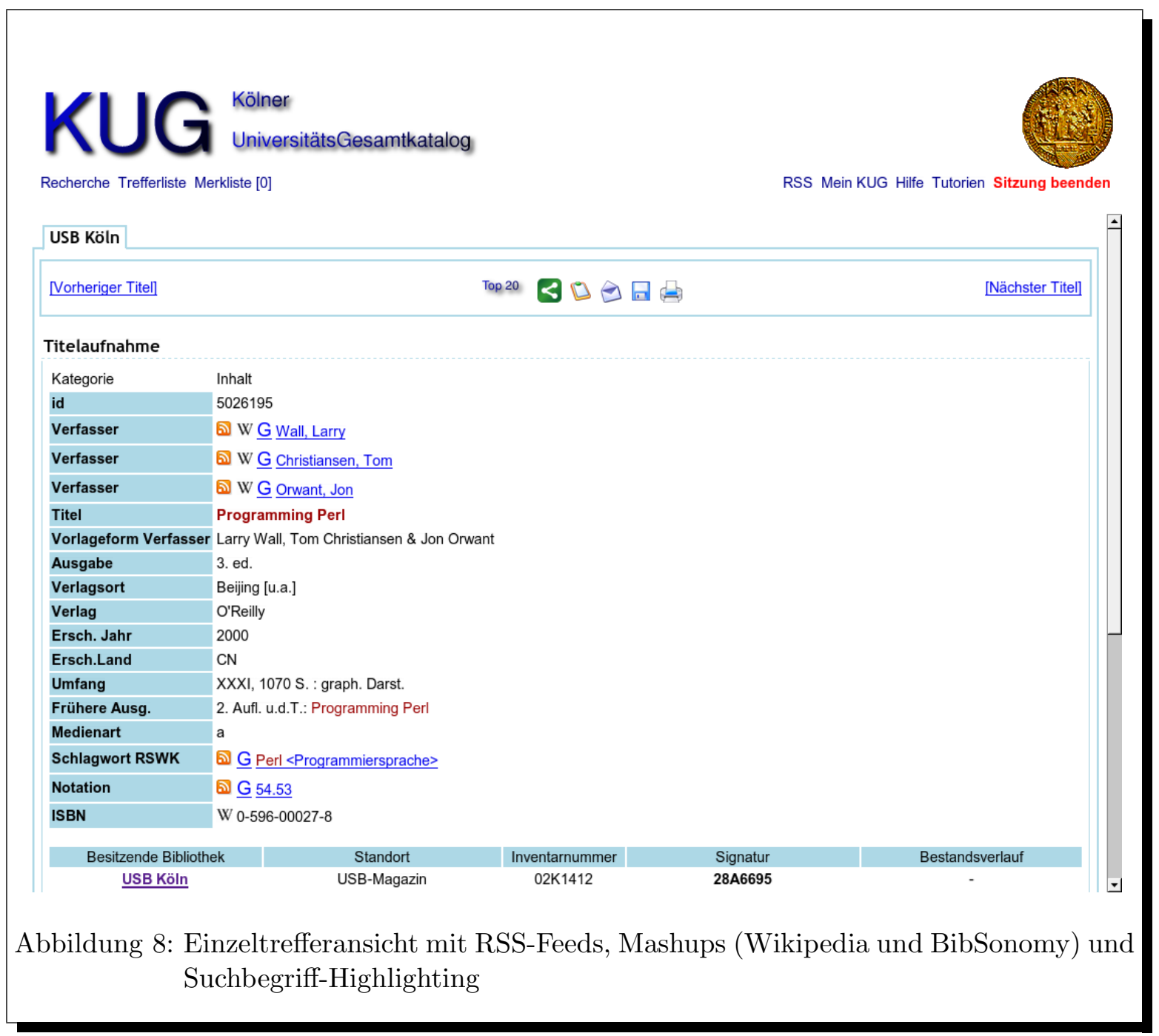

Umgekehrt lässt sich der KUG auch selbst (nach Absprache) über externe webbasierte Zugriffsschnittstellen in externe Recherche-Portale über Mashups einbinden. So sind die Bestände des 
KUG derzeit z.B. in die Digitale Bibliothek NRW (DigiBib) und UK-Online ${ }^{28}$ eingebunden.

Zur unmittelbaren Recherche im Portal über einen Web-Browser steht ein Such-Plugin für den Browser Firefox zur Verfügung, das ausgehend von der KUG-Hilfeseite installiert werden kann.

\section{Die Zukunft von OpenBib}

Mit den schon erreichten Funktionen von OpenBib und dem KUG ist die Weiterentwicklung speziell in Richtung OPAC 2.0 - noch nicht abgeschlossen.

Für die Weiterentwicklung von OpenBib sind folgende Bereiche vorgesehen:

\subsection{Teilhabe der Nutzer am OPAC}

Auch hier zeigen andere Online-Plattformen wie Amazon oder im Bibliotheksbereich der Karlsruher XOPAC, wie Benutzer so eingebunden werden können, dass sie selbst einen Mehrwert für andere Nutzer und damit letztlich auch für die jeweilige Anwendung schaffen können. In diesen Bereich gehören Ratings, Kommentare sowie Weiterentwicklungen des schon vorhandenen Taggings durch Nutzer. So können z.B. ausgehend vom Tagging-Verhalten eines einzelnen Nutzers zusätzlich „Nachbarschaften“ zu anderen Nutzern ermittelt und ein Zugriff auf deren Tag-Sammlungen ermöglicht werden.

Für die so geschaffenen Informationen von Nutzern für Nutzer muss auch überlegt werden, wie sie zwischen verschiedenen Recherche-Portalen frei ausgetauscht bzw. generell genutzt werden können (z.B. über WebServices). So ein Austausch würde sich auch für Informationen anbieten, die durch dezentrale Nutzungsanalysen gewonnen werden, wie z.B. RecommenderInformationen. Derzeit bietet die UB Karlsruhe mit BibTin ${ }^{29}$ bereits einen Recommenderdienst für die Integration in externe Onlinekataloge an.

\subsection{Weitere Mashups}

Neben den bereits realisierten Mashups können weitere externe Dienste integriert werden. Ein Beispiel in diesem Bereich ist Geolokalisierung, wie sie schon der Dreiländerkatalog des hbz verwirklicht. Darüber hinaus soll der Mashup mit BibSonomy dahingehend erweitert werden, dass auch dort vergebene Tags und Verknüpfungen zu anderen Titeln für OpenBib erschlossen werden können.

\footnotetext{
${ }^{28}$ uk-online - Hochschul-Kommunikationssystem der Universität zu Köln. http://uk-online.uni-koeln.de/ (Zugriff am 30.3.2007)

${ }^{29}$ BibTip - Ein Recommendersystem für Onlinekataloge. http://www.bibtip.de/ (Zugriff am 13.4.2007)
} 


\subsection{Suchverfeinerung}

Für die Orientierung in Ergebnislisten werden in OpenBib schon verschiedene Verfahren angewendet. In diesem Bereich soll nach weiteren Optimierungen der bereits realisierten Drilldowns gesucht werden. Ebenso muss analysiert werden, wie sich Klassifikationsbäume auf Treffermengen sinnvoll einbinden lassen.

\subsection{Weitere Recherche-Backends}

Neben der Einbindung alternativer Suchmaschinen-Backends, wie z.B. Lucene oder KinoSearch, stellt sich die Frage, wie sich Datenbestände einbinden lassen, bei denen kein Vollzugriff auf die Daten besteht. Hier bietet sich nur die Möglichkeit, sie über entsprechende AbfrageStandards wie Z39.50, SRU usw. in der Form weiterer Recherche-Backends einzubinden. Damit fallen jedoch automatisch auch alle bereits dargestellten Vorteile weg, die sich aus einer einheitlichen lokalen Datenhaltung ergeben. Bei lizenzpflichtigen Angeboten kann das aber der einzige Weg sein.

\section{Fazit}

Der KUG mit OpenBib und der neue Karslruher OPAC mit XOPAC zeigen, wie mit OpenSource-Software innovative Wege im Bibliotheksbereich beschritten werden können. Der Weg ist sicherlich noch weit, aber die ersten Schritte wurden gemacht. 


\section{Kurz-Biographie des Autors}

Oliver Flimm, Jahrgang 1969, ist Diplom-Physiker und als Administrator sowie Programmierer im Bereich Unix/Linux seit 1991 tätig. Während seines Studiums der Physik mit Nebenfach Informatik arbeitete er an der USB Köln und betreute dort technisch das Projekt InstitutsGesamtKatalog (IGK). Nach Abschluss seines Studiums übernahm er dort als wissenschaftlicher Mitarbeiter neben seiner Tätigkeit im Bereich Unix/Linux-Administration und -Programmierung u.a. die technische Durchführung verschiedener Projekte - insbesondere des KUG-Projektes.

\section{Anschrift des Autors}

Oliver Flimm

Universitäts- und Stadtbibliothek Köln

Dezernat Datenverarbeitung

Universitätsstr. 33

D-50931 Köln

E-Mail: flimm@ub.uni-koeln.de

\section{Lizenz}

Der Artikel steht unter der Creative Commons CC-BY Lizenz 